\title{
Communiquer avec les familles : une compétence trop rarement enseignée
}

\author{
Communication with patients' families: yet a rarely taught skill among physicians in the intensive \\ care units
}

\author{
La Commission Jeunes de la Société de Réanimation de Langue Française*
}

Reçu le 19 août 2013 ; accepté le 27 août 2013

(C) SRLF et Springer-Verlag France 2013

Les symptômes anxieux, dépressifs ou de stress posttraumatique sont fréquents chez les proches des patients de réanimation [1-3]. Des travaux réalisés lors de la dernière décennie ont montré que certaines techniques de communication (livrets, entretiens proactifs) peuvent diminuer l'incidence de ces symptômes [4-6]. Néanmoins, la prise en charge des familles n'est, à notre connaissance, que rarement l'objet d'enseignements spécifiques.

La Commission Jeunes de la Société de Réanimation de Langue Française (SRLF) a mené une enquête, disponible en ligne sur le site de la SRLF de juin à septembre 2012, sur les pratiques de communication entre les soignants et les proches des malades de réanimation. Les 764 répondants avaient en moyenne 38 ans (écart-type : dix ans) et étaient des médecins avec plus de deux ans d'expérience de post-internat pour $45 \%$ d'entre eux. Ils travaillaient dans des services dont près de la moitié n'étaient ouverts aux visites des proches que moins de six heures par jour. Un quart des services était ouvert $24 \mathrm{~h} / 24$. Un livret d'accueil destiné aux proches était disponible dans $87 \%$ des services des répondants. Des procédures écrites concernant la communication avec les familles avaient été établies dans $13 \%$ des services des participants.

Parmi les $20 \%$ de répondants connaissant des échelles d'évaluation de l'anxiété/dépression des familles, seule la

La Commission Jeunes de la Société de Réanimation de Langue Française* $(\bowtie)$

Société de Réanimation de Langue Française,

Maison de la Réanimation,

48, avenue Claude Vellefaux, F-75010 Paris

e-mail : commissionjeunessrlf@gmail.com

* Pierre-Marie BERTRAND, Alexandra BEURTON, Alice BLET (secrétaire), Benjamin CLOUZEAU, Laetitia CONTENTIN, Damien CONTOU, Pierre-Éric DANIN, Bruno FILLOUX, Sylvain GARNIER, Élodie GELISSE, Antoine GROS, Vincent PEIGNE (secrétaire adjoint), Nicolas de PROST, Alexis SOUMMER et Florent WALLET moitié d'entre eux les utilisaient parfois. Seulement $40 \%$ des répondants avaient la possibilité de travailler avec un psychologue attaché à leur service : $28 \%$ leur adressaient des familles de façon ponctuelle et $5 \%$ de façon systématique.

Plus de la moitié des répondants rapportaient avoir déjà vécu des situations conflictuelles lors d'entretien. De même, $56 \%$ des participants disaient se sentir gênés par le comportement ou les questions de familles plusieurs fois par an. À peine $20 \%$ des répondants avaient assisté à une formation sur les aspects relationnels avec les familles. L'accès à ce type de formation était considéré comme difficile pour $75 \%$ des participants alors que ces formations étaient perçues comme utiles de façon presque unanime (95\%). L'intervention, dans ces formations, de spécialistes non médicaux était souhaitée par $86 \%$ des soignants interrogés. Le recours à des méthodes d'enseignement avec mise en situation (simulation, jeux de rôle) semblait intéressant pour $75 \%$ des participants.

La prise en charge des familles durant le séjour du patient est ainsi devenue une composante incontournable de l'activité clinique des réanimateurs. Il existe un réel besoin de développer et de diffuser des enseignements permettant d'acquérir une excellence dans ce domaine.

Au-delà de l'amélioration de la compréhension de la situation clinique par les familles et de la diminution de l'incidence des symptômes anxieux et dépressifs, une meilleure communication pourrait également permettre de diminuer la fréquence des conflits entre les familles et les soignants.

\section{Références}

1. Pochard F, Azoulay E, Chevret S, et al (2001) Symptoms of anxiety and depression in family members of intensive care unit patients: ethical hypothesis regarding decision-making capacity. Crit Care Med 29:1893-7 
2. Azoulay E, Pochard F, Kentish-Barnes N, et al (2005) Risk of post-traumatic stress symptoms in family members of intensive care unit patients. Am J Respir Crit Care Med 71:987-94

3. Van Pelt DC, Milbrandt EB, Qin L, et al (2007) Informal caregiver burden among survivors of prolonged mechanical ventilation. Am J Respir Crit Care Med 175:167-73

4. Azoulay E, Pochard F, Chevret S, et al (2002) Impact of a Family Information Leaflet on Effectiveness of Information Provided to Family Members of Intensive Care Unit Patients. A multicenter, prospective, randomized, controlled trial. Am J Respir Crit Care Med 165:438-42

5. Lautrette A, Darmon M, Megarbane B, et al (2007) A communication strategy and brochure for relatives of patients dying in the ICU. N Engl J Med 356:469-78

6. Kinnersley P, Edwards A, Hood K, et al (2007) Interventions before consultations for helping patients address their information needs. Cochrane Database of Systematic Reviews 2007: CD004565 